\title{
Invasive Haemophilus influenzae type b disease in the Oxford region (1985-91)
}

\author{
Robert Booy, Sara A Hodgson, Mary P E Slack, Eileen C Anderson, \\ Richard T Mayon-White, E Richard Moxon
}

\begin{abstract}
For a seven year period (1985-91) clinical and epidemiological data were prospectively collected on children aged $<10$ years with microbiologically confirmed invasive Haemophilus influenzae type b infection in the Oxford region to study the epidemiology of the disease and determine the potential impact of early primary immunisation in infants. Computer records of primary immunisations given to these cases were retrospectively analysed and, where necessary, hospital and general practitioner records were searched to determine the immunisation history.
\end{abstract}

Over the seven year period, 416 cases of invasive $H$ influenzae type $b$ disease were reported. Widescale immunisation against $H$ influenzae type b began in 1991 as part of a regional trial. The estimated annual incidence for invasive disease between 1985 and 1990 was 35.5 cases per 100000 children aged less than 5 years; for $H$ influenzae type b meningitis it was $25 \cdot 1$ per 100000 children aged less than 5 years. The cumulative risks for invasive disease and meningitis by the fifth birthday were one in 560 and one in 800 respectively. The majority of disease $(71 \%)$ occurred in children less than 2 years of age with the peak monthly incidences at 6 and 7 months of age. The overall mortality was $4.3 \%$ and $50 \%$ of these deaths occurred suddenly. Most $(91 \%)$ of the children had received at least one primary immunisation against diphtheria, tetanus, and pertussis before $H$ influenzae type $b$ infection and there was only one case of parental refusal of immunisation. None had received $H$ influenzae type b immunisation. Given a vaccine uptake of $90 \%$ by 5 months of age it is estimated that at least $82 \%$ of the $H$ influenzae type $b$ infections could have been prevented. Extrapolated nationally, 1150 cases of infection and 50 deaths could be prevented each year by routine primary immunisation.

(Arch Dis Child 1993; 69: 225-228)

Haemophilus influenzae type b is a major cause of serious bacterial infection in early childhood. Throughout the 1980 s and early 1990 s this organism has consistently been the most frequent cause of bacterial meningitis in children reported from microbiology laboratories in England and Wales. ${ }^{1}$
Infants are protected for the first few months after birth by maternal antibodies and to a lesser extent by breast feeding. ${ }^{2}{ }^{3}$ However, the peak incidence of disease occurs later in the first year when the concentration of antibodies against the type $b$ capsule, polyribisol ribitol phosphate (PRP), is at its lowest. ${ }^{2}$

The first effective vaccine consisted of purified PRP but was only protective in children older than 18 months of age. ${ }^{4}$ Conjugate vaccines are now available in which PRP, or an oligosaccharide derived from it, is covalently linked to a carrier protein. Trials have shown protection of infants from as young as 2 months of age, ${ }^{56}$ except for one study in Alaska where a conjugate vaccine of lesser immunogenicity failed to provide significant protection in a population known for its high incidence of early infection. ${ }^{7}$ This vaccine was highly protective in Finland where the median age of infection is much older. ${ }^{8}$ Variation in the epidemiology of invasive $H$ influenzae type $\mathrm{b}$ disease may profoundly affect the efficacy of vaccination.

We report the results of seven years of prospective epidemiological surveillance of invasive $H$ influenzae type $\mathrm{b}$ disease in the Oxford region performed in preparation for a prospective study of $H$ influenzae type b conjugate vaccine efficacy that began in the first half of 1991. For a vaccine to be effective, it has to reach those at risk. To predict what impact there might be from the planned introduction of routine immunisation against $H$ influenzae type $\mathrm{b}$ in the $\mathrm{UK}$, we have ascertained the history of routine immunisation against diphtheria, tetanus, and pertussis in children who have had $H$ influenzae type b disease.

\section{Methods}

Since 1 January 1985, an intensive epidemiological survey of bacteriologically confirmed invasive $H$ influenzae type b disease in children $<10$ years of age has been performed with the active cooperation of all consultant microbiologists in the Oxford region. A case was defined either by isolation of the organism from a normally sterile site such as blood or cerebrospinal fluid, or by a positive $H$ influenzae type $\mathrm{b}$ antigen test from cerebrospinal fluid or joint fluid together with a positive gram stain result.

A brief clinical report as well as the isolated strain were sent to the haemophilus reference laboratory at the Oxford Public Health Laboratory, where serotyping and antibiotic
Correspondence to: Dr Booy.

Accepted 24 March 1993 
sensitivity were confirmed. Isolates from children who have died outside hospital have been included where postmortem evidence was consistent with an invasive $H$ influenzae type $b$ infection.

To test the completeness of data collection, cases were cross referenced with the Oxford Record Linkage database (1985-91) and Communicable Disease Reports (1990-1). Also, clinical data such as date of birth, survival, and age at presentation were confirmed where necessary by reviewing hospital notes. All cases admitted to hospitals in the Oxford region were included even if only temporarily resident, but Oxford residents admitted to hospitals outside the region were excluded. Taking 1988 as the mid-year of the survey, the geographically defined denominator population was 2518000 of whom 171800 were $<5$ years of age according to projections from the Office of Population Censuses and Surveys.

In addition the routine immunisation history of cases of $H$ influenzae type $\mathrm{b}$ infection was ascertained from district immunisation computer records. Where necessary further details were obtained from hospital inpatient records or by contacting the family doctor.

Both the epidemiological survey and the study to check routine immunisation history were approved by the central Oxford research ethics committee.

The $\chi^{2}$ test was used in the comparison of ampicillin resistance of isolates in 1985-7 with 1988-90. Fisher's exact test was used to compare mortality associated with ampicillin resistant strains with that seen with ampicillin sensitive strains.

\section{Results}

Over the seven year survey period, a total of 416 cases of invasive $H$ influenzae type b disease were ascertained in children $<10$ years of age. All but six were reported through our intensive microbiological surveillance; four extra cases were identified from the Oxford Record Linkage Study and two from Communicable Disease Reports.

Only $7 \%$ of isolates were not reviewed at the haemophilus reference laboratory in Oxford; this was because the isolate died in transit or was not sent. However, these cases were all microbiologically confirmed at the admitting hospital. The exact age at presentation was known in 415 cases of which 405 were $<5$

Clinical presentations by age of 415 cases of $\mathrm{H}$ influenzae type $b$ disease in children in the Oxford region, fanuary 1985-December 1991

\begin{tabular}{|c|c|c|c|c|c|}
\hline & \multicolumn{5}{|c|}{ Age (years) } \\
\hline & $<1.0$ & $1.0-1.9$ & $2 \cdot 0-4 \cdot 9$ & $\geqslant 5 \cdot 0$ & $\begin{array}{l}\text { No (\%) } \\
\text { total }\end{array}$ \\
\hline Meningitis & 139 & 82 & 63 & 5 & $289(70)$ \\
\hline Epiglottitis & 4 & 11 & 29 & 4 & 48 (12) \\
\hline Cellulitis & 18 & 8 & 2 & 0 & $28(7)$ \\
\hline \multicolumn{6}{|l|}{ Bone/joint } \\
\hline infection & 9 & 5 & 7 & 0 & $21(5)$ \\
\hline Bacteraemia & 6 & 5 & 4 & 0 & $15(4)$ \\
\hline Pneumonia & 2 & 1 & 4 & 1 & $8(2)$ \\
\hline Other & 3 & 2 & 1 & 0 & $6(1)$ \\
\hline
\end{tabular}

years of age $(98 \%)$ and $295<2$ years $(71 \%)$. Disease incidence was highest in those $<1$ year of age and the peak monthly incidence occurred jointly at 6 and 7 months of age (figure). However, only 24 cases $(6 \%)$ were $<4$ months of age. Also only 12 cases (3\%) occurred between 4 and 5 years of age. The clinical presentations of these cases by age is shown in the table.

A yearly average of 63 cases occurred from $1985-90(57,54,68,75,58$, and 65 respectively) but only 39 occurred in 1991 when immunisation against $H$ influenzae type b began as part of a large scale trial. This lower figure is significantly less than previous years $(p<0.01)$. The vaccine was available in three of eight districts from May 1991 (and another from September) but only to infants $<6$ months of age, and even with high uptake only two infections (in children $<10$ years of age) were predicted to have been prevented by the end of 1991. The percentage of 1991 infections in children $<1$ year of age was not reduced compared with previous years, that is in 1991 there were fewer cases in children of all ages.

The estimated annual incidences for invasive $H$ influenzae type $\mathrm{b}$ disease and $H$ influenzae type $\mathrm{b}$ meningitis between 1985 and 1990 were respectively 35.5 and $25 \cdot 1$ per 100000 children aged $<5$ years. Thus the cumulative risks for invasive disease and meningitis by the fifth birthday were $1: 560$ and 1:800 respectively.

In keeping with similar surveys, 910 infection was more common in the cooler months with $53 \%$ of cases occurring in the five months from October to February.

Overall, boys were more commonly affected than girls $(1 \cdot 33: 1)$ and this held true separately for meningitis $(1 \cdot 20: 1)$, epiglottitis $(1 \cdot 40: 1)$, and a combination of the other infections $(1 \cdot 17: 1)$.

There have been 18 deaths: 14 resulting from meningitis, three from epiglottitis, and one from bacteraemia, giving an overall mortality of $4.3 \%$ and a mortality for meningitis of $4.8 \%$. The age range of deaths was 2 months -5 years with a median of 15 months. Only two infants were aged $<5$ months. The

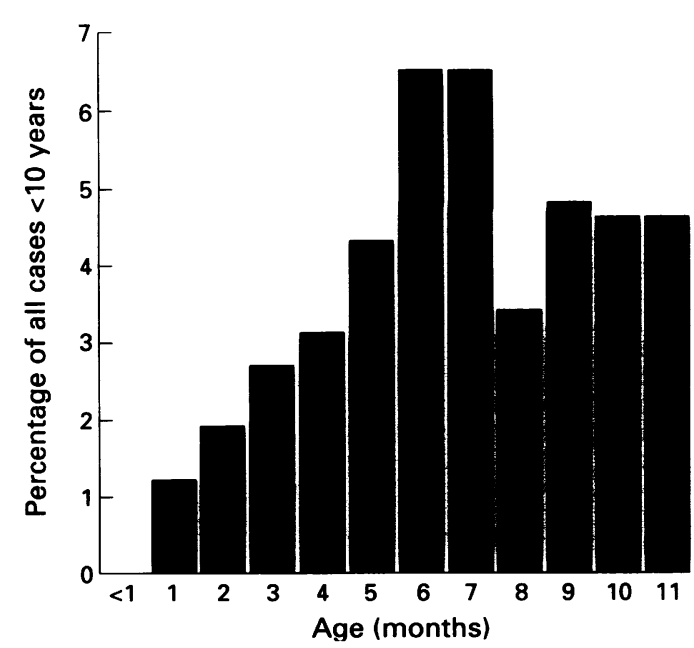

Distribution of $\mathrm{H}$ influenzae type $b$ disease by age in months of the 181 cases occurring in the first year of life. 
male:female ratio of deaths was $2: 1$. Nine $(50 \%)$ of the deaths occurred suddenly with the child either being found dead at home or in cardiorespiratory arrest on arrival in casualty.

Ampicillin resistance, secondary to $\beta$ lactamase production, was found in $12.3 \%$ of isolates. None was chloramphenicol resistant. There was no significant difference in the rate of ampicillin resistance on comparison of isolates from 1985-7 with those from 1988-90 $(p>0.3)$. The case fatality rate was similar whether or not an isolate was resistant to ampicillin: $6 \%$ versus $4 \%(p>0 \cdot 3)$.

The history of primary immunisation against diphtheria, tetanus, and pertussis was determined in 401 (96\%) children with invasive $H$ influenzae type b disease. Virtually all of these children were immunised according to the three, five, and nine month schedule as individual districts in the Oxford region (there are eight in all) did not adopt the accelerated two, three, and four month schedule until either 1991 or 1992 . The number (percentage) of children who received at least one, two, or three primary immunisations before infection were $364(91 \%), 321(80 \%)$, and $235(59 \%)$ respectively. Of the 37 children not immunised before infection, 27 subsequently received primary immunisations, three died from the illness so could not be immunised, in six the subsequent immunisation history could not be ascertained, and in only one case was it recorded on the computer files that parents had refused primary immunisation. Fifteen of the 18 deaths $(83 \%)$ had received at least one, and 13 at least two, primary immunisations before death. Two infants were aged between 2 and 3 months so had not yet started primary immunisation and the third died at 6 months without being immunised.

\section{Discussion}

The findings presented are consistent with, but expand upon, previously published data on the first 200 cases observed in this prospective survey. ${ }^{11} \mathrm{H}$ influenzae type $\mathrm{b}$ is a common and serious infection of early childhood. It has however received far less public attention than meningococcus group B partly because that organism causes periodic outbreaks and perhaps also because it affects teenagers and adults as well as children.

It was reassuring to find from cross reference with the Oxford Record Linkage database and recent data from Communicable Disease Reports that relatively complete data collection has been achieved in this special survey. The calculated incidence of invasive infection, however, represents a minimal estimate since adequate cultures are not always collected and even when they are, previous antibiotic treatment may prevent recognition of the pathogen.

The incidence observed is remarkably similar to a recent report from Wales. ${ }^{9}$ A somewhat lower incidence of disease has been estimated from an extended UK survey that began in 1990 and encompasses, in addition to Oxford and Wales, four other English regional health authorities. ${ }^{12}$ However, it is known that the degree of under reporting has been greater in the other English regions than in the Oxford region (personal communication, C MacLeod). As the annual live birth rate in the UK is about 790000 , we estimate that 1400 cases of invasive $H$ influenzae type b disease and over 900 cases of $H$ influenzae type b meningitis occur annually in UK children aged $<5$ years.

The significantly lower incidence in 1991 is most likely due to natural fluctuation. It was too early to be a direct vaccine effect as only two cases were predicted to have been prevented by the end of 1991 . It was unlikely to be an indirect effect either as there was an equivalent reduction in vaccine and control districts $(43 \% v 32 \%)$. Furthermore, vaccine was only offered to children $<6$ months of age and yet there was an equal reduction in cases among those $<1$ year of age compared with those $>1$ year. Nationally, the number of laboratory reports was similar in 1990, 1991, and $1992(1210,1138 \text {, and 1088) })^{13-15}$ which argues against a widespread secular trend. Under reporting was also unlikely as there was enthusiastic cooperation from district laboratories and cross referencing with both the Oxford Record Linkage Study and Communicable Disease Reports showed that on average fewer than one case per year was missed in our survey.

Resistance to ampicillin by $H$ influenzae type b has not changed significantly over the study period and nor does ampicillin resistance appear to contribute to the mortality associated with infection. Although we have not observed chloramphenicol resistance in children within the survey period, one case was found in an adult in 1991 and one case occurred in a child in 1992. The results of this survey support the use of empiric combination antibiotic treatment with ampicillin and chloramphenicol, although the emergence of chloramphenicol resistance raises the question of whether third generation cephalosporin treatment may become more appropriate.

Recent studies have shown very high levels of protection afforded by $H$ influenzae type $\mathrm{b}$ conjugate immunisation even after only two doses in early infancy. 56 Our data show that among cases of $H$ influenzae type b disease, $80 \%$ had received at least two routine primary injections before illness and $91 \%$ at least one injection. Remembering that these infants had been immunised according to an expanded three, five, and nine month schedule, it can be inferred that at least $90 \%$ of infants could have received two or more injections before their illness, had an accelerated two, three, four month schedule of immunisation against $H$ influenzae type $\mathrm{b}$ been in operation.

The potential value of the new accelerated schedule is further highlighted by the observations that (i) the peak months for $H$ influenzae type $b$ infection in our survey were 6 and 7 months of age, (ii) of the small number of cases who did not receive primary immunisation before illness the great majority were subsequently immunised, and that (iii) only $59 \%$ of cases had received three primary immunisations (on the previous schedule) before 
illness. Estimates derived in 1992 from computer records of childhood immunisation in Oxfordshire suggest that the average age of children completing their third immunisation according to the accelerated schedule is $<5$ months (personal communications, D Howells). If 5 months is taken as a cut off for receiving three injections, then $82 \%$ of the $H$ influenzae type $b$ infections we have seen could have been prevented. This is based on the observation that $91 \%$ of cases occur in infants $\geqslant 5$ months (see figure) and assumes vaccine uptake of $90 \%$ and efficacy of $100 \%$ for three doses of $H$ influenzae type b conjugate vaccine. An uptake figure similar to this has already been observed in the Oxford study of H influenzae type $b$ conjugate vaccine efficacy which began in 1991, so even higher uptake could be attainable in routine practice. The average age at which primary immunisation has been completed in study infants was $<5$ months. Furthermore as of 1 October 1992, no cases of disease have occurred in Finnish or Oxford regional children receiving two or more doses of PRP-T vaccine ${ }^{16}$ (personal communication, $\mathrm{J}$ Eskola). Extrapolating these estimates to the UK, 1150 cases of infection could be prevented each year.

As the use of $H$ influenzae type b conjugate vaccine can dramatically reduce oropharyngeal carriage $^{17}$ thereby lowering the risk of transmission, it is likely that even greater protection can be achieved by a $90 \%$ vaccine uptake rate.

The potential value of the accelerated schedule is also seen in that the great majority of the observed deaths ( $89 \%$ ) have occurred in infants over 5 months of age, and most fatal cases $(83 \%)$ had received at least one primary immunisation before illness. The high rate $(50 \%)$ of deaths occurring suddenly further underlines the value of immunoprophylaxis. An $83 \%$ reduction in mortality would equate to 50 lives saved each year in the UK.

Catch-up immunisation for children aged up to 4 years is recommended in the UK. This will provide protection for the great majority of children at risk as only $3 \%$ of cases occur between 4 and 5 years of age and a lesser percentage thereafter.

Among children infected by $H$ influenzae type $b$ we found only one case of complete refusal of immunisation. The uptake of the new $H$ influenzae type $b$ vaccine may be maximised if health professionals can educate parents about the seriousness of $H$ influenzae type $b$ disease and also about the efficacy and safety of immunisation.

We are especially grateful to the consultant microbiologists of the Oxford region, Dr J B Selkon, Dr D Crooke, Dr J V Dadswell, Dr A R Stacey, Dr M McIntyre, Dr D A Leigh, Dr D Waghorn, Dr C Hall, Dr R A Cox, Dr R G Masterton, Dr B C Das, Dr A P Gillett, and Dr M Severn for supporting this study. The cooperation of immunisation coordinators, Dr G Sleight, The cooperation of immunisation coordinators, Dr G Sleight, Mrs J Moreton, Dr E Haworth, Dr D Lessing, Dr M Kiddle, Dr
$S$ Shribman, Dr V Fernandez, Dr S Holton, and Dr C Mlynek and support staff throughout the region has been much appreciated. We also thank Dr Michael Goldacre for his help in making available data from the Oxford Record Linkage study and Dr Norman Begg for providing data from Communicable Disease Reports.

1 Office of Population Censuses and Surveys. Communicable disease statistics, statistical tables, 1982-90. London: HMSO, 1982-90.

2 Fothergill $L D$, Wright J. Influenzal meningitis: the relation of age incidence to the bactericidal power of blood against the causal organism. f Immunol 1933; 24: 273-84.

3 Cochi SL, Fleming DW, Hightower AW, et al. Primary invasive Haemophilus influenzae type $b$ disease: a population-based assessment of risk factors. $\mathcal{F}$ Pediatr 1986; 108: 887-96.

4 Peltola H, Kayhty H, Virtanen M, Makela PH. Prevention of Haemophilus influenzae type $\mathrm{b}$ bacteremic infections with the capsular polysaccharide vaccine. $N$ Engl $\mathcal{f}$ Med 1984; 310: 1561-6.

5 Black SB, Shinefield HR, Fireman B, et al. Efficacy in infancy of oligosaccharide conjugate Haemophilus influenzae type b (HbOC) vaccine in a United States population of 61,080 children. Pediatr Infect Dis 71991 ; population $97-104$

6 Santosham $M$, Wolff $M$, Reid $R$, et al. The efficacy in Navajo infants of a conjugate vaccine consisting of Haemophilus influenzae type $b$ polysaccharide and Neisseria meningitidis outer membrane protein complex. N Engl F Med 1991; 324: 1767-72.

7 Ward J, Brenneman G, Letson GW, Heyward WL. Limited efficacy of a Haemophilus influenzae type b conjugate vaccine in Alaska native infants. N Engl f Med 1990; 323: 1393-401.

8 Eskola J, Peltola H, Takala AK, et al. Efficacy of Haemophilus influenzae type b polysaccharide-diphtheria toxoid conjugate vaccine in infancy. $N$ Engl $\mathcal{F}$ Med 1987; 317: 717-22

9 Howard AJ, Dunkin KT, Musser JM, Palmer SR. Epidemiology of Haemophilus influenzae type $\mathrm{b}$ invasive disease in Wales. BMf 1991; 303: 441-5.

10 Takala AK, Eskola J, Peltola H, Makela PH. Epidemiology of invasive Haemophilus influenzae type $b$ disease among children in Finland before vaccination with Haemophilus influenzae type b conjugate vaccine. Pediatr Infect Dis $\mathcal{F}$ 1989; 8: 297-302.

11 Tudor-Williams G, Frankland J, Isaacs D, et al. Haemophilus influenzae type $\mathrm{b}$ disease in the Oxford region. Arch Dis Child 1989; 64: 517-9.

12 Nazareth B, Slack MPE, Howard AJ, Waight PA, Begg NT. A survey of invasive Haemophilus influenzae infections. Communicable Disease Report 1992; 2: R13-6.

13 Public Health Laboratory Service. Communicable Disease Report 1991; 1: 9.

14 Public Health Laboratory Service. Communicable Disease Report 1992; 2: 7 .

15 Public Health Laboratory Service. Communicable Disease Report 1993; 3: 3 .

16 Booy R, Moxon ER, Macfarlane JA, Mayon-White RT, Slack MPE. Efficacy of Haemophilus influenzae type b conjugate vaccine in Oxford region. Lancet 1992; 340: 847 .

17 Takala AK, Eskola J, Leinonen $\mathrm{M}$, et al. Reduction of oropharyngeal carriage of Haemophilus influenzae type $b$ (Hib) in children immunized with an Hib conjugate vaccine. $f$ Infect Dis 1991; 164: 982-6. 